\title{
Sulfa Allergy: Cross-Reactivity Versus Multiple Concurrent Allergies
}

\author{
${ }^{1}$ Muhammad Tariq Shakoor, ${ }^{2}$ Samia Ayub and ${ }^{3}$ Zunaira Ayub \\ ${ }^{1}$ Resident Physician, Internal Medicine Department, St Mary Mercy Hospital, Livonia, MI, USA \\ ${ }^{2}$ Resident Physicain, Internal Medicine Department, Mount Auburn Hospital, Cambridge, MA, USA \\ ${ }^{3}$ Medical Student, Fatima Jinnah Medical College, Lahore, Pakistan
}

Received 2012-10-13, Revised 2013-05-01; Accepted 2013-12-02

\begin{abstract}
As a medical resident we have always been taught that there is some sort of cross reactivity between sulfonamide antibiotics and nonantibiotic sulfonamides. Even the manufacturer's package inserts contain a precautionary statement about possible Cross-reactivity. The most common approach to this problem is avoidance of all sulfa containing drugs. However, there are few data supporting this contraindication. Thus we may be withholding appropriate therapies from patients unnecessarily. To provide a critical and comprehensive review of literature to explore either cross reactivity between sulfonamide antibiotics and nonantibiotic sulfonamides is a fact or fiction and to present an approach to use nonantibiotic sulfonamides in sulfa allergic patients. A PubMed and general medline search was conducted using the individual names of nonantibiotic sulfonamides. We reviewed all of the available case reports and studies regarding sulfonamide antibiotic cross-reactivity with nonantibiotic sulfonamides. Also reviewed the manufacturer's package insert for each nonantibiotic sulfonamide drug for information concerning possible cross-reactivity with sulfonamide antibiotics. Sulfa drug allergy is one word holding the whole cross-reactivity theory in it. It should be obsolete from the medical dictionary. This one word is complicating the medical decision-making. Allergies should not be attributed to classes or groups of drugs unless proven. After reviewing all the available literature we can conclude that assumptions about cross-reactivity are a FICTION.
\end{abstract}

Keywords: Non-Antibiotic, Cross-reactivity, Contraindication, Sulfonamides, Sulfonamide Antibiotics

\section{INTRODATION}

Sulfonamides are commonly used drugs in primary care practice. Reactions to Sulfonamide Antibiotics (SA) are relatively common as compared to other antimicrobials. They occur in approximately $3 \%$ of the treatment courses; however, only $3 \%$ of these are true hypersensitivity reactions. Two types of allergic reactions have been described in relation to these compounds. One being an IgE mediated maculopapular or urticarial rash developing 1-3 days after medication initiation and the other being a hypersensitivity reaction. The hypersensitivity reaction, consisting of fever and non-urticarial rash, usually develops 7-14 days after the medication initiation (Johnson et al., 2005). The term "sulfa" refers to a derivative of an antimicrobial agent, sulfanilamide. The term has been applied to a diverse group of drugs, all of which contain the sulfonamide chemical structure; - $\mathrm{SO} 2 \mathrm{NH} 2$ moiety which is also a part of many common medications, such as thiazide and loop diuretics (Johnson et al., 2005). We can classify sulfa drugs in two ways, one is based on the chemical structure i.e., sulfonylartylamine, non sulfonylarylamine and sulfonamide moiety containing drugs (Fig. 1) (Johnson et al., 2005) and the other is based on cross reactivity theory i.e., Sulfonamides Antibiotics (SA) and Sufonamides Non-Antibiotics (SNA) (Table 1A) (Strom et al., 2003).

Corresponding Author: Muhammad Tariq Shakoor, Resident Physician, Internal Medicine Department, St Mary Mercy Hospital, Livonia, MI, USA 
Sulfonylarylamine includes antibiotics e.g sulfanilamide, sulfamethoxazole etc.

Non-sulfonylarylamine includes CAIs, thiazides, loop diuretics, sulfonylureas, COX-2 inhibitors, protease inhibitors etc.

Sulfonamide moiety includes sumatriptan, naratriptan, topiramate, zonisamide, ibutilide, sotalol, probenecid etc.

Fig. 1. Classification of sulfonamides, $\mathrm{CAI}=$ carbonic anhydrase inhibitors; COX-2 $=$ cyclooxygenase-2; thiazides $=$ thiazide diuretics and related compounds

Table 1A. Sulfa drugs

\begin{tabular}{|c|c|c|}
\hline Antibiotic sulfonamide & Subgroups & $\begin{array}{l}\text { Drugs } \\
\text { Sulfadiazine, sulfisoxazole } \\
\text { sulfamethoxazole, sulfapyridine }\end{array}$ \\
\hline \multirow[t]{7}{*}{ Non antibiotic sulfonamide } & Carbonic anhydrase inhibitors & $\begin{array}{l}\text { Acetazolamide, Brinzolamide, } \\
\text { Dichlorphenamide, Dorzolamide, } \\
\text { Methazolamide, Sulthiame }\end{array}$ \\
\hline & Loop diuretics & $\begin{array}{l}\text { Bumetanide, Furosemide, } \\
\text { Piretanide, Torsemide }\end{array}$ \\
\hline & Thiazides and related compounds & $\begin{array}{l}\text { Bendroflumethiazide, Benzthiazide, } \\
\text { Chlorothiazide, Chlorthalidone, } \\
\text { Clopamide, Diazoxide, } \\
\text { hydrochlorothiazide, } \\
\text { Hydroflumethiazide, Indapamide, } \\
\text { methyclothiazide, metolazone, } \\
\text { Polythiazide, Xipamide }\end{array}$ \\
\hline & Sulfonylureas & $\begin{array}{l}\text { Acetohexamide, Chlorpropamide } \\
\text { Gliclazide, Glibornuride, Glipizide, } \\
\text { Glimepiride, Gliquidone, Glyburide, } \\
\text { Glymidine, Tolazamide, Tolbutamide }\end{array}$ \\
\hline & $\begin{array}{l}\text { Cyclooxygenase } 2(\mathrm{COX}-2) \text { inhibitors } \\
\text { protease inhibitors }\end{array}$ & $\begin{array}{l}\text { Celecoxib, rofecoxib, valdecoxib } \\
\text { Amprenavir, Fosamprenavir }\end{array}$ \\
\hline & Triptans & Naratriptan, Sumatriptan \\
\hline & Other agents & $\begin{array}{l}\text { Amprenavir, Dapsone, } \\
\text { Fosamprenavir, Ibutilide, } \\
\text { Probenecid, Sotalol, Sulfasalazine, } \\
\text { Topiramate, Zonisamide }\end{array}$ \\
\hline
\end{tabular}

As a medical resident we have always been taught that there is some sort of cross-reactivity between SA and SNA. Even the manufacturer's package insert contains a precautionary statement about possible crossreactivity (Table 1B). The most common approach to this problem is avoidance of all sulfa containing drugs. However, there is handful data supporting this contraindication, thus we may be withholding appropriate therapies from patient unnecessarily (Ditto, 2002; Knowles et al., 2001; SDISCPN, 2003). 
Muhammad Tariq Shakoor et al. / American Journal of Infectious Diseases 9 (4): 148-154, 2013

Table 1B. Manufacturer's package insert information for sulfonamide-containing medications

\begin{tabular}{|c|c|c|c|}
\hline \multirow[b]{2}{*}{ Drug Class } & \multicolumn{2}{|c|}{ Manufacturer's package insert statement } & \multirow[b]{2}{*}{ Brand } \\
\hline & Presence & Recommendation & \\
\hline \multicolumn{4}{|l|}{ Carbonic anhydrase inhibitors } \\
\hline acetazolamide & Yes & Warnings & Avkare \\
\hline brinzolamide & yes & Warnings & Azopt \\
\hline Dichlorphenamide & no & & Daranide \\
\hline dorzolamide & yes & Warnings & Cosopt \\
\hline methazolamide & yes & Warnings & Methazolamide \\
\hline Sulthiame & yes & Contraindications & Ospolot \\
\hline \multicolumn{4}{|l|}{ Loop diuretics } \\
\hline bumetanide & yes & Warnings & Bumetanide \\
\hline furosemide & yes & Precautions & Furosemide \\
\hline Piretanide & yes & Contraindications & Tauliz \\
\hline torsemide & no & & Demadex \\
\hline \multicolumn{4}{|c|}{ Thiazide diuretics and related compounds } \\
\hline Bendroflumethiazide & yes & Contraindications & Corzide \\
\hline Benzthiazide & yes & Contraindications & Aquatag \\
\hline Chlorothiazide & yes & Contraindications & Diuril \\
\hline chlorthalidone & yes & Contraindications & Thalitone \\
\hline Clopamide & yes & Contraindications & Brinaldix \\
\hline diazoxide & yes & Contraindications & Hyperstat \\
\hline hydrochlorothiazide & yes & Contraindications & Hydrochlorothiazide \\
\hline Hydroflumethiazide & yes & Contraindications & Saluron \\
\hline indapamide & yes & Contraindications & Indapamide \\
\hline methyclothiazide & yes & Contraindications & Enduronyl \\
\hline metolazone & yes & Warnings & Mykrox \\
\hline Polythiazide & yes & Contraindications & Renese \\
\hline Xipamide & yes & Contraindications & Aquatag \\
\hline \multicolumn{4}{|l|}{ Sulfonylureas } \\
\hline Acetohexamide & no & & Dymelor \\
\hline chlorpropamide & no & & Diabenese \\
\hline Glibornurinde & no & & Glutril \\
\hline Gliclazide & no & & Diamicron \\
\hline Glipizide & no & & Glipizide \\
\hline Glimepiride & no & & Amaryl \\
\hline Gliquidone & no & & Glurenorm \\
\hline Glyburide & no & & Diabeta \\
\hline Glymidine & no & & Redul \\
\hline Tolazamide & no & & Tolinase \\
\hline Tolbutamide & no & & Orinase \\
\hline \multicolumn{4}{|l|}{ 5-HT3 receptor antagonists } \\
\hline Naratriptan & no & & Amerge \\
\hline Sumatriptan & no & & Imitrex \\
\hline \multicolumn{4}{|l|}{ COX-2 inhibitors } \\
\hline Celecoxib & yes & Contraindications & Celebrex \\
\hline Rofecoxib & no & & Vioxx \\
\hline Valdecoxib & yes & Contraindications & Bextra \\
\hline \multicolumn{4}{|l|}{ Other compounds } \\
\hline Amprenavir & yes & Precautions & Agenerase \\
\hline Dapsone & no & & Aczone \\
\hline Fosamprenavir & yes & Precautions & Lexival \\
\hline Ibutilide & no & & Corvert \\
\hline Probenecid & no & & Probenicid \\
\hline Sotalol & no & & Sorine \\
\hline Sulfasalazine & yes & Contraindications & Azulfidine \\
\hline Topiramate & no & & Topamax \\
\hline Zonisamide & yes & Contraindications & Zonegran \\
\hline
\end{tabular}




\subsection{Data Sources}

A PubMed and general medline search was conducted using the individual names of non-antibiotic sulfonamides (48 non-antibiotic sulfonamides were identified). Terms like hypersensivity, adverse effects and cross- reactions were used. We reviewed all the available case reports and studies about sulfonamide antibiotic cross-reactivity with non-antibiotic sulfonamides. A critical review of the methodology, results and conclusion for each available study was conducted. Review of the manufacturer's package insert for each nonantibiotic sulfonamide drug was performed to obtain information concerning possible cross-reactivity with sulfonamide antibiotics (Table 1B).

\subsection{Literature Review}

The cross reactivity hypothesis was tested in a large retrospective cohort study by Strom et al. (2003) Authors identified 20,226 patients seen from 1987 through March 1999 who were prescribed a systemic SA and then at least 60 days later received a SNA. Researchers reviewed records to determine whether patients described as having an allergic reaction to SA were at increased risk of having a subsequent allergic reaction to SNA of 969 patients with an allergic reaction after a SA, $96(9.9 \%)$ had an allergic reaction after receiving a SNA. For comparison, out of 19,257 patients who had no allergic reaction after a SA, $315(1.6 \%)$ had an allergic reaction after receiving a SNA (adjusted odds ratio, 2.8; 95\% confidence interval, 2.1 to 3.7 ).

However, risk of an allergic reaction was greater after receiving penicillins, in patients with a prior hypersensitivity reaction to $\mathrm{SA}$, as compared to patients with no such history (adjusted odds ratio, 3.9; 95\% confidence interval, 3.5 to 4.3). Furthermore, among patients who had prior hypersensitivity reaction to a SA, risk of an allergic reaction after the subsequent receipt of a SNA was lower than the risk of an allergic reaction after the subsequent receipt of a penicillin (adjusted odds ratio, 0.7; $95 \%$ confidence interval, 0.5 to 0.9 ). Similarly, it was seen that in patients with a history of hypersensitivity to penicillins, risk of an allergic reaction after the receipt of a SNA was lower than the SA (adjusted odds ratio, 0.6; 95\% confidence interval, 0.5 to 0.8 ).

Hence, the data shows that among SA-allergic patients, the incidence of concurrent penicillin allergy was greater than the incidence of reactivity to SNA. This observation seems to imply an element of cross-reactivity between SA and penicillins, which is biochemically a distinct group. The incidence of allergic reactions to SNA drugs was higher among penicillin allergic individuals than among SA allergic patients. As it is not possible to suggest crossover sensitivity between penicillins and SNA, the authors conclude that simultaneous allergy to SA and SNA is common and likely exclusive of cross-reactivity.

In a small retrospective study Lee and co-researchers revealed small risk of cross-reaction. Researchers reviewed 363 hospital charts examined 34 patients with a self-reported history of sulfa allergy who were subsequently given acetazolamide, furosemide, or both (Lee et al., 2004). The nature of the self-reported sulfa allergic reaction was documented in $79 \%$ of the 34 patients. Of these, 329 patients $(91 \%)$ were excluded of the remaining 34 cases that did report so-called sulfa allergy, 13 (38\%) received acetazolamide alone, 7 (21\%) received furosemide alone and $14(41 \%)$ received both acetazolamide and furosemide of the 27 patients who received acetazolamide, 10 (37\%) had no documented allergic cross-reaction to sulfa and $2(7 \%)$ cases had urticaria. None of the patients experienced severe allergic cross-reaction to sulfa of 21 patients who received furosemide, no unpredictable adverse reactions or allergic cross-reactions to sulfa was noted. They concluded that there is little clinical or pharmacological evidence to suggest that a self-reported sulfa allergy is likely to produce a life-threatening cross-reaction with acetazolamide or furosemide. They also concluded that these medications should be considered if the risk-tobenefit ratio warrants their use.

Patterson et al. (1999) evaluated literature associated with several sulfonamide drug classes including Carbonic Anhydrase Inhibitors (CAI), sulfonylureas, loop diuretics, thiazide diuretics, 5-HT agnonists and COX-2 inhibitors. Authors performed three meta-analyses and looked at data from 14 randomized controlled trials involving 11008 patients. In the first meta-analysis, patients with a reported allergy to sulfonylarylamines and non-sufonylarylamines, such as furosemide, thiazide diuretics or sulfonylureas, who received celecoxib $(\mathrm{n}=$ $73)$, a placebo $(\mathrm{n}=32)$ or an active comparator $(\mathrm{n}=32)$ were included. There was no statistically significant difference among the 3 groups in terms of potential allergic events. In the end of the three meta-analyses the incidence of allergic reactions with celecoxib was compared to the active comparators. No statistically significant findings were noted in these meta-analyses. Based on these findings authors concluded that the risk of cross-reactivity between SA and celecoxib is low. Based on this review Johnson et al. (2005) made the recommendation that patients with a sulfonylarylamine allergy can be administered medications from the other two groups with proper monitoring if it is not possible to use an unrelated product. 
On the other hand Hamstreet and Page followed patients during their hospital stay to document prescription and adverse reactions of SA and SNA (Hemstreet, 2006). Inpatient and outpatient use of potentially cross-reactive drugs was observed in $52 \%$ of patients, $60 \%$ of those patients took furosemide. No adverse effects were reported or documented with outpatient or inpatient SNA use, even among patients with a history of life-threatening reactions to SA.

All of the above studies conclude that patients with a history of allergic reactions to drugs may be at increased risk for all drug-induced adverse events that appear to be allergic in nature. Practically this crossreactivity theory is not valid.

Now let's see if theoretically cross-reactivity is possible. Brackett et al. (2004) and coworkers concluded that the difference in chemical structure between the sulfonylarylamines and other types of sulfonamides implies that cross-reactivity is unlikely. However, T-cell mediated immune response to the unmetabolized, nonhaptenated parent SA has been reported to occur occasionally, although it is not known whether T-cell recognition of antibiotic is related to the sulfonamide functional group or not. Until the mechanism of this recognition was elucidated, cross-reactivity with SNA appeared to remain theoretically possible (Slatore and Tilles, 2004). In 2007 the same first author Brackett concluded that the stereo specificity of T-cell response renders cross-reactivity between SA and SNA highly unlikely (Stock, 1999). Apparently cross-reactivity responses to sulfonamide-containing drugs likely represent multiple concurrent, rather than linked, drug hypersensitivities. Hence, most up to date researchers conclude that the cross-reactivity can neither be explained theoretically nor practically (Brackett, 2007).

Finally, in an exhaustive review of all the relevant case reports from 1955 to 2004 Johnson et al. (2005) concluded that, none of the drug pairs implicated during this 50-year period showed enough strength of association to establish the existence of sulfonamide cross-reactivity.

In total 48 sulfonamide non-antibiotics were identified during the search listed in Table 1A. We listed all the important information in the Manufacturer's Package Inserts (MPI), in Table 1B. When we analyzed all of these 48 MPI together, 21 MPI documented no reaction; rest of the $27 \mathrm{MPI}$ documented the risk of cross-reactivity under contraindications (18), warnings (6) and precautions (3). Six Carbonic Anhydrase Inhibitors (CAIs) were identified. The MPI for all CAI contains the warning and contraindication except dichlorphenamide, whereas they have also mentioned that fatalities have occurred, although rarely, due to severe reactions to SA. We identified four sulfonamide loop diuretics during search. Their MPI recommends some sort of reaction with SA except torsemide. Torsemide MPI lists a contraindication to sulfonylurea use, whereas none of the MPI for sulfonylureas shows any concern regarding concurrent use of torsemide. Thirteen thiazide diuretics and related compounds were identified. All of these medications list SA allergy either in contraindications or in warnings. Eleven sulfonylureas and two 5HT3 receptor blocking drugs were identified and none of the MPI includes any statement concerning their use in SA allergy. We identified three COX-2 inhibitors. Only celecoxib and valdecoxib MPI contain a statement concerning their use in SA allergy, whereas this group generated the largest number of studies concerning their cross-reactivity with SA and all concluded that there is no cross-reactivity (Patterson et al., 1999). Nine drugs were identified in miscellaneous group. Only amprenavir, fosamprenavir, sulfasalazine and zonisamide MPI have cautionary statements. The protease-inhibitors are sulfonamides with an N4 arylamine, like the sulfonylarylamine antibiotics. The product labeling for amprenavir and fosamprenavir state that the potential for cross-sensitivity with other sulfonamides is unknown, but they should be used with caution in people with SA allergy.

\section{DISCUSSION}

The story started in 1955 when Mosely and Baroody (1955) reported that a patient with congestive heart failure and sulfa allergy experienced neurological side effects following administration of oral acetazolamide. Upon readministration of acetazolamide patient experienced same neurological symptoms accompanied by respiratory distress and limb edema. Authors concluded that patient experienced hypersensitive reaction to acetazolamide and since acetazolamide is chemically related to SA; patient with sulfonamide allergy should not receive acetazolamide. As mentioned by the authors neurological symptoms were related to hypokalemia. Stock (1999) interpreted this case as being inconclusive stating that the second episode could have been due to congestive heart failure exacerbation.

Most of the data regarding cross-reactivity theory consist of case reports suggesting that patients who are allergic to sulfonylarylamines are at an increased risk of allergic reaction to the other sulfonamide group medications (Hemstreet, 2006; Landor and Rosenstreich, 
1993; Bretza, 1982; Hansbrough et al., 1987). However, the allergic response to two different drugs may represent either true cross-allergenicity, or an independent immune response to each drug. According to Sullivan (1991), multiple, independent drug allergies are quite common and patients who are allergic to one antimicrobial drug have at least a 10 -fold increased risk of reacting to another structurally unrelated drug. Clinical presentation of immunologically mediated reactions is very diverse, hypersensitivity response to structurally similar drugs cannot automatically be attributed to cross-allergenicity. This way, case reports develop a body of evidence but are unable to discriminate immunologically linked reactions from concurrent unrelated drug hypersensitivities.

The co-existence of hypersensitivity reactions to several drugs does not prove cross-reactivity between them. As concluded by Strom et al. (2003) patients with a prior sulfa allergy are even more likely to have an allergic reaction to penicillin; a structurally unrelated drug, than they are to a SNA. Additionally, the risk of an allergic reaction after receiving a SNA was higher in patients with a history of penicillin allergy than in those with a history of hypersensitivity to SA. One should understand that there is a marked difference between cross-reactivity and the likelihood of mounting an immune response to a wide range of haptens.

Most of the available evidence suggests that SNA need not to be routinely avoided in patients with a history of allergy to SA (Strom et al., 2003; Johnson et al., 2005; Brackett et al., 2004). SNA may cause an allergic reaction themselves due to stereo specificity with sulfonylarylamines, cross-reactivity though is unlikely (Brackett et al., 2004). Unfortunately, the product labeling of many SNA do not correlate with what is known scientifically. For instance, many diuretics are either contraindicated or contain warnings regarding their use in patients with a history of allergic reaction to SA (Table 1B).

This confliction with the product information of drugs confuses many medical practitioners and pharmacists. In another study authors studied the knowledge and attitudes of American pharmacists concerning sulfonamide allergy cross-reactivity (Wall et al. 2010). They suggested significant diversity in knowledge and attitudes of pharmacists concerning cross-reactivity of SA and SNA.

We can end this discussion with the conclusion that there is no theoretical or practical evidence to support cross-reactivity between SA and SNA. However, the clinicians must remain alert to the possibility of multiple and severe drug sensitivities in patients who experienced an allergic response to a SA. Ultimately a clinician needs to make the decision of whether to initiate a sulfa drug in SA allergic patient, on a risk to benefit basis.

\section{CONCLUSION}

To corroborate the cross-sensivity reaction of SA and SNA, we did not find any strong documentation in literature supporting this reaction. All of the reported cases of reactions, on close inspection, do not provide any cause-effect relationship. Although case reports describe numerous apparently linked hypersensitivity reactions to SA and SNA, no investigation to date has identified a plausible mechanism for such cross-reactivity.

After reviewing all the available literature we conclude that sulfa drug allergy label is misleading, it is a very vague medical terminology. Sulfa drug allergy is a misnomer. It should be obsolete from the medical dictionary. We are withholding appropriate therapies from patients without any evidence and we are complicating the medical decision making.

Now the question is can we use SNA in SA sensitive patients? The best answer is "probably yes" because susceptible individuals also have a much greater incidence of concurrent allergy to other drugs and drug classes. Administering first dose under supervision, close monitoring, starting with low dose and patient counseling of the potential problem and benefit of the therapy in high risk patients should be employed during the initial stage of therapy. Also it is vital to recognize the allergic reactions correctly, not documenting common side effects of medications like nausea, vomiting, diarrhea, photo dermatitis, as allergic reactions. As a general principle, all allergic adverse reactions to medications should be recorded in the patient's file with the specific name of the drug or drugs to which the patient has reacted and the nature of the reaction. Allergies should not be attributed to classes or groups of drugs unless proven.

\section{REFERENCES}

Brackett, C.C., 2007. Sulfonamide allergy and crossreactivity. Curr. Allergy Asthma Rep., 7: 41-48. DOI: $10.1007 / \mathrm{s} 11882-007-0029-8$

Brackett, C.C., H. Singh and J.H. Block, 2004. Likelihood and mechanisms of cross-allergenicity between sulfonamide antibiotics and other drugs containing a sulfonamide functional group. Pharmacotherapy: J. Hum. Pharmacol. Drug Therapy, 24: 856-870. DOI: 10.1592/phco.24.9.856.36106 
Bretza, J.A., 1982. Thrombocytopenia due to sulfonamide cross-sensitivity. Wis. Med. J., 81: 2123. PMID: 7113249

Ditto, A.M., 2002. Drug Allergy. A. Introduction, Epidemiology, Classification of Adverse Reactions, Immunochemical Basis, Risk Factors, Evaluation of Patients with Suspected Drug Allergy, Patient Management Considerations. In: Patterson's Allergic Diseases, Grammer, L.C. and P.A. Greenberger (Eds.), Lippincott Williams and Wilkins, Philadelphia, ISBN-10: 0781723868, pp: 295-334.

Hansbrough, J.R., H.J. Wedner and D.D. Chaplin, 1987. Anaphylaxis to intravenous furosemide. J. Allergy Clin. Immunol., 80: 538-541. DOI: 10.1016/00916749(87)90004-2

Hemstreet, B.A., 2006. Sulfonamide allergies and outcomes related to use of potentially cross-reactive drugs in hospitalized patients. Pharmacotherapy, 26: 551-557. PMID: 16553515

Johnson, K.K., D.L. Green, J.P. Rife and L. Limon, 2005. Sulfonamide cross-reactivity: Fact or fiction? Ann. Pharmacother, 29: 290-301. DOI: 10.1345/aph.1E350

Knowles, S., L. Shapiro and N.H. Shear, 2001. Should celecoxib be contraindicated in patients who are allergic to sulfonamides? Drug. Saf., 24: 239-247. DOI: $10.2165 / 00002018-200124040-00001$

Landor, M. and D.L. Rosenstreich, 1993. Vesiculobullous rash in a patient with systemic lupus erythematosus. Ann. Allergy, 70: 196-203. PMID: 8452312

Lee, A.G., R. Anerson, R.H. Kardon and M. Wall, 2004. Presumed "sulfa allergy" in patients with intracranial hypertension treated with acetazolamide or furosemide: Cross-reactivity, myth or reality? Am. J. Ophthalmol., 138: 114-118. DOI: 10.1016/j.ajo.2004.02.019
Mosely, V. and N.B. Baroody, 1955. Some observations on the use of acetazoleamide ("Diamox") as an oral diuretic in various edematous states and in uremia with hyperkaliemia. Am. Pract. Dig. Treat., 6: 558566. PMID: 14361946

Patterson, R., A.E. Bello and J. Lefkowith, 1999. Immunologic tolerability profile of celecoxib. Clin. Ther., 21: 2065-2079. DOI: 10.1016/S01492918(00)87238-0

SDISCPN, 2003. Sulfonamide cross-reactions explained. Saskatchewan Drug Information Services College of Pharmacy and Nutrition.

Slatore, C.G. and S.A. Tilles, 2004. Sulfonamide hypersensitivity. Immunol. Allergy Clin. North Am., 24: 477-490. DOI: 10.1016/j.iac.2004.03.011

Stock, J.G., 1990. Sulfonamide hypersensitivity and acetazolamide. Arch. Ophthalmol., 108: 34-35. DOI: 10.1001/archopht.1990.01070070020005

Strom, B.L., R. Schinnar, A.J. Apter, D.J. Margolis and E. Lautenbach et al., 2003. Absence of crossreactivity between sulfonamide antibiotics and sulfonamide nonantibiotics. N. Engl. J. Med., 349: 1628-1635. DOI: 10.1056/NEJMoa022963

Sullivan, T.J., 1991. Cross-reactions among furosemide, hydrochlorothiazide and sulfonamides (letter). JAMA, 265: 120-121. DOI: 10.1001/jama.265.1.120 Wall, G.C., J.E. Dewitt, S. Haack, A. Fornoff and D.K. Eastman et al., 2010. Knowledge and attitudes of American pharmacists concerning sulfonamide allergy cross-reactivity. Pharm. World Sci., 32: 343346. DOI: 10.1007/s11096-010-9389-6 